\title{
MIEDO EN CONTEXTOS DE EDUCACIÓN FÍSICA, ACTIVIDAD FÍSICA Y DEPORTE: REVISIÓN NARRATIVA (2010-2019)
}

\author{
FEAR IN CONTEXTS OF PHYSICAL EDUCATION, PHYSICAL \\ ACTIVITY AND SPORT: NARRATIVE REVIEW (2010-2019) \\ MEDO NOS CONTEXTOS DE EDUCAÇÃo FÍSICA, ATIVIDADE \\ FÍSICA E ESPORTE: REVISÃO NARRATIVA (2010-2019)
}

Felipe Nicolás Mujica-Johnson ${ }^{1}$

\section{Resumen}

En este artículo de revisión narrativa se analiza los sentidos subjetivos del miedo de las personas que participan en contextos de educación física, actividad física y deporte. Se indagó en ocho bases de datos que permitieron seleccionar 32 estudios empíricos realizados con un método cualitativo. La elección de los estudios respondió a los criterios establecidos en torno a la metodología de investigación, la fecha de publicación, los participantes y su contenido. Dichos estudios fueron sometidos a un análisis de contenido que incluyó el programa informático de análisis de datos cualitativos Atlas.ti 7.5. Los resultados presentan cuatro categorías centrales: (a) incompetencia motriz, (b) errores y dificultades en el desempeño motriz, (c) daño y sufrimiento corporal y (d) discursos culturales. Finalmente, se concluye que existe la necesidad de promover acciones pedagógicas de educación afectiva que permitan abordar al profesorado y al alumnado el miedo en dichos contextos. Asimismo, se aprecia que es fundamental promover el respeto a la dignidad humana para prevenir situaciones de violencia que, en diferentes ocasiones, son atribuciones causales de temor.

Palabras clave: violencia; afectividad; educación moral; educación física; deporte

\section{Abstract}

This narrative review article analyzes the subjective senses of fear of people who participate in contexts of physical education, physical activity and sport.. It was investigated in eight databases that allowed to select 32 empirical studies carried out with a qualitative method. The choice of studies responded to the criteria established around the research methodology, the date of publication, the participants and their content. These studies underwent a content analysis that included the Atlas.ti 7.5 qualitative data analysis software. The results present four central categories: (a) motor incompetence; (b) errors and difficulties in motor performance; (c) bodily harm and suffering; and (d) cultural speeches. Finally, it is concluded that there is a need to promote pedagogical actions of affective education that will address teachers and students fear in these contexts. It is also appreciated that it is essential to promote respect for human dignity to prevent situations of violence that, on different occasions, are causal attributions of fear.

Keywords: violence; affectivity; moral education; physical education; sport

1 Doctorando en Ciencias de la Actividad Física y del Deporte, Universidad Politécnica de Madrid, Madrid, España. Facultad de Ciencias de la Actividad Física y del Deporte-INEF, Universidad Politécnica de Madrid, Madrid, España. Correo electrónico: fmujica@live.cl. Orcid: 0000-0002-6956-2357. 


\section{Resumo}

Este artigo de revisão narrativa analisa os sentidos subjetivos do medo das pessoas que participam dos contextos de educação física, atividade física e esporte. Foi investigado em oito bases de dados que permitiram selecionar 32 estudos empíricos realizados com método qualitativo. A escolha dos estudos respondeu aos critérios estabelecidos em torno da metodologia da pesquisa, data de publicação, participantes e seu conteúdo. Esses estudos foram submetidos a uma análise de conteúdo que incluiu o programa qualitativo de análise de dados Atlas.ti 7.5. Os resultados apresentam quatro categorias centrais: (a) incompetência motora; (b) erros e dificuldades no desempenho motor; (c) dano e sofrimento corporal; e (d) discursos culturais. Por fim, conclui-se que é necessário promover ações pedagógicas de educação afetiva que abordem o medo de professores e alunos nesses contextos. Também é apreciado que é essencial promover o respeito à dignidade humana para evitar situações de violência que, em diferentes ocasiões, são atribuições causais do medo.

Palavras chave: violência; afetividade; educação moral; Educação Física; esporte

Para citar este artículo:

Mujica Johnson, F. N. (2019). Miedo en contextos de educación física y deporte: revisión narrativa (20102019). Lúdica Pedagógica, 30, 71-81. https://doi.org/10.17227/ludica.num30-11106 


\section{INTRODUCCIÓN}

Los contextos de actividad física y deporte, son espacios en que las emociones suelen vivenciarse continuamente, debido a que sus participantes involucran la totalidad de su corporeidad, de modo que son sensibles a pensamientos, acciones de las demás personas o a valoraciones externas. Por lo anterior, es posible entender la actividad físico-deportiva como un proceso desafiante a nivel emocional, donde la persona se implica con el mundo de manera activa, permitiéndole re-significarlo (Hurtado, 2008). 0 sea, en aquel proceso psicofísico (Husserl, 1962; Mujica, 2020; Stein, 2007), existe una percepción subjetiva del cuerpo, la cual permite al ser humano establecer vínculos emocionales con el entorno (González y González, 2010). Precisamente, las emociones se suelen asociar a significados subjetivos, construidos y re-construidos en forma sistemática durante las experiencias de vida, ya que tienen un contenido histórico-cultural (Forde, 2013; González, 2008; 2009; Mujica-Johnson y Jiménez, 2019).

La idea que las emociones son dotadas socialmente de significado, y que sus formas de expresión cambian según el contexto sociocultural, es sostenida por cognitivistas, sociólogos y antropólogos (Bolaños, 2016; Lazarus, 2000; Rebollo y Hornillo, 2010; Zaccagnini, 2008), lo cual impulsa a atribuir mayor importancia a los sentidos subjetivos durante este tipo de actividades (González, 2002; 2009). Así, dicho sentido se entiende en este estudio como "la unidad inseparable de las emociones y de los procesos simbólicos" (González, 2008, p. 233). En concreto, este estudio se enfoca en el miedo, de modo que en el siguiente apartado se abordará algunos aspectos teóricos de dicha emoción.

\section{Aspectos teóricos en torno al miedo}

El miedo, ha de entenderse como una sensación ante elementos que significan una amenaza real o percibida (Consuegra, 2010). Por tanto, cumpliría funciones vitales para el ser humano. En concreto, como mecanismo de defensa, instinto de conservación y fuerza primitiva, ya que alertaría al organismo para enfrentarse a peligros (Huber, 2000). Entonces, se consideraría normal, incluso saludable que una persona vivencie el miedo en una situación real de peligro. No obstante, percibir esa emoción se torna problemático cuando, en contextos seguros, afecta negativamente la práctica de actividad física y/o deportiva y, por cierto, la participación en educación física (EF, en adelante).

Desde una perspectiva sociológica, se ha señalado que el miedo encuentra sus raíces en aspectos culturales. En concreto, en discursos de poder, de género, de discriminación y, por cierto, de violencia (Beltrán-Carrillo, Devís-Devís y Peiró-Velert, 2016; Stewart, Gray, Kelly y Maclsaac, 2019). En este sentido, Monforte y Pérez-Samaniego (2017) señalan que "el miedo es una experiencia situada en contextos relacionales concretos, por lo que el estudio de sus significados y consecuencias no puede separarse de la comprensión de dichos contextos" (p. 86).

Por otra parte, desde una mirada pedagógica, el miedo ha sido atribuido a las relaciones interpersonales que se producen en contextos formales o informales. Justamente, porque suele ser considerado como una emoción negativa para el proceso educativo, ya que es reconocido por perturbar, obstaculizar e incluso llegar a invalidar el proceso de aprendizaje (López, 2016). Por consiguiente, resulta imprescindible entender que el miedo puede producirse por múltiples motivos y, por ello, es necesario comprenderlos para favorecer el aprendizaje en contextos de actividad física y deporte (Barker, Nyberg y Larsson, 2019; Canales-Lacruz y Rey-Cao, 2014; Salvador-García, Chiva-Bartoll y Vergaz, 2018). Igualmente, el miedo actuaría como barrera para la actividad física en contextos de salud pública, de modo que es fundamental aplicar estrategias que lo reduzcan en pacientes con diferentes enfermedades (Curbelo, Zarco, Almodóvar, Flórez y Carmona, 2017; Gay, Eschalier, Levyckyj, Bonnin y Coudeyre, 2018; Vader et al., 2017; Ziebart et al., 2018).

Con base en lo mencionado anteriormente, este estudio de revisión tuvo por objetivo analizar los sentidos subjetivos del miedo en las personas en los contextos de EF, actividad física y deporte.

\section{METOdOLOGÍA}

El diseño del estudio es una revisión narrativa, basada en una búsqueda exhaustiva de la información (Mujica, Orellana y Concha, 2017). La revisión narrativa evalúa la información a partir de biblio- 
grafía especializada sobre la temática, y se considera apropiada para la descripción y discusión del estado del arte de un determinado asunto, desde el punto de vista teórico o contextual (Rother, 2007). En la presente investigación la revisión se enfoca en estudios recientes que aborden la dimensión cognitiva del miedo, describiendo las atribuciones y significancias subjetivas que lo hayan originado. La investigación se centra en los momentos señalados por Merello y Oliva (2012), que son (a) planteamiento de la problemática, (b) búsqueda exhaustiva de la información a partir de buscadores y bases de datos, (c) análisis de la literatura y (d) redacción de los resultados de acuerdo con los objetivos planteados.

La investigación se enfoca en la siguiente pregunta de búsqueda: ¿cuáles son los sentidos subjetivos del miedo por parte de las personas que participan en contextos de EF y deporte? Los criterios de inclusión para la selección de artículos fueron los siguientes: (a) estudios empíricos en un contexto vinculado a la $\mathrm{EF}$, la actividad física o al deporte, realizados con un diseño metodológico cualitativo y en cualquier idioma. Publicaciones en inglés y español; (b) se utilizaron estudios de ambos sexos y de todas las edades; (c) artículos disponibles en texto completo, y (d) publicaciones realizadas desde el 1 de enero del 2010 al 16 de diciembre del 2019. Las bases de datos consultadas han sido Google Académico, Red de Revistas Científicas de América Latina y el Caribe, España y Portugal (REDALYC), Red Iberoamericana de Innovación y Conocimiento Científico (REDIB), Dialnet, Scientific Electronic Library Online (scielo), Directory of Open Access Journals (DOAJ), Scopus y Web of Science. Los términos utilizados para la búsqueda son miedo, educación física, actividad física y deporte. De esta manera, 32 estudios de carácter científico fueron seleccionados, los cuales se presentan con mayor detalle en la tabla 1, donde además se incluyen los datos de su contexto y temática.

Tabla 1. Estudios seleccionados para la revisión narrativa

\begin{tabular}{|c|c|l|}
\hline N.o de estudios & \multicolumn{1}{|c|}{ Contexto } & \multicolumn{1}{c|}{ Contenido temático } \\
\hline 15 & EF & $\begin{array}{l}\text { Danza; Fútbol; Voleibol; Expresión corporal; Condición } \\
\text { Física; Ciclismo; Parkour; TEA; Método AICLE; Corporeidad y } \\
\text { género; Competencia motriz; Acoso escolar; Dodgeball }\end{array}$ \\
\hline 8 & Actividad Física y Salud & $\begin{array}{l}\text { Espondiloartritis; VIH; Osteoporosis; Osteoartritis de } \\
\text { rodilla; Lesión medula espinal; Obesidad; Riesgo social en la } \\
\text { infancia; Reconstrucción del ligamento cruzado anterior. }\end{array}$ \\
\hline 4 & Formación Docente EF & Expresión corporal; Baloncesto; Natación \\
\hline 5 & Deporte & $\begin{array}{l}\text { Corporeidad y género; Fútbol, género y raza; } \\
\text { Deportes extremos; Balonmano; Baloncesto }\end{array}$ \\
\hline
\end{tabular}

EF: Educación Física. AICLE: Aprendizaje Integrado de Contenidos y Lengua Extranjera. TEA: Trastorno del Espectro Autista. VIH: Virus de Inmunodeficiencia Humana.

Fuente: Elaboración propia a partir de la búsqueda bibliográfica.

Los datos recogidos de los estudios fueron analizados en un nivel semántico desde la perspectiva cualitativa por medio de un análisis de contenido (Taylor y Bogdan, 2009). El procedimiento del análisis fue leer en forma detenida y sistemáticamente los estudios, seleccionando las citas vinculadas al objetivo del estudio (sentidos subjetivos del miedo) para luego ser codificadas, produciendo un refinamiento de los datos para alcanzar la comprensión del tema de estudio. Para optimizar la codificación, relación y teoriza- ción de los datos, se utilizó el programa informático Atlas.ti, versión 7.5, reconocido por su alcance en el análisis cualitativo (Mujica-Johnson y Jiménez, 2019).

\section{RESULTADOS}

Los sentidos subjetivos del miedo en los contextos de EF y deporte se presentan en cuatro categorías: (a) incompetencia motriz, (b) errores y dificultades en el desempeño motriz, (c) daño y sufrimiento cor- 
poral y (d) discursos culturales. La figura 1 refleja las relaciones semánticas que existen entre estas categorías, las cuales se han construido a partir de los hallazgos recogidos en los estudios seleccionados para la revisión narrativa.

Figura 1. Relaciones semánticas del miedo en contextos de EF, actividad física y deporte Fuente: Elaboración propia a partir del análisis de contenido en el software Atlas.ti 7.5.

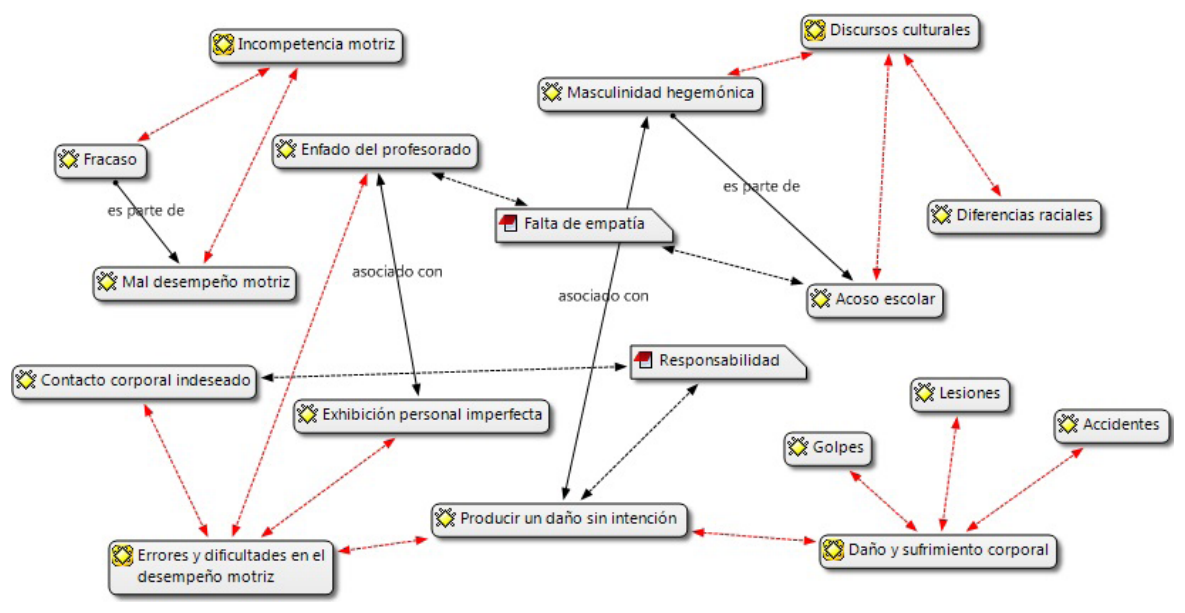

\section{Incompetencia motriz}

La experiencia motriz que cada persona ha acumulado en su trayectoria personal de actividad física o deporte influye en su competencia motriz (Ruiz y Graupera, 2003), al igual que en su autopercepción física (Hellín, Moreno y Rodríguez, 2006), lo cual explica que la falta de práctica físico-deportiva en escolares haya dificultado el afrontamiento en las tareas para el desarrollo de la condición física en la clase de EF, produciéndoles miedo a tener un mal desempeño, a fracasar en sus metas personales $\mathrm{y}$, por consecuencia, temer lograr una baja calificación (Mujica, Orellana, Aránguiz y González, 2016). Esta dificultad de afrontamiento de las tareas también generó que en el aprendizaje de voleibol en la clase de EF una alumna sintiera miedo ante la ausencia de un logro cercano al ideal motriz (Monforte y Pérez-Samaniego, 2017). En términos similares, un estudio en un centro educativo de Alemania revela la asociación del miedo con la competencia motriz del alumnado de secundaria (Leisterer y Jekauc, 2019). Las expectativas de fracaso y la mala competencia motriz percibida pueden ser potenciadas a partir de este tipo de experiencias valoradas como desagradables, ya que el cerebro humano las almacena en la memoria episódica como recuerdos emocionales (Boyano, 2012).
Los nexos realizados entre cognición, emoción y objetivos, generan recuerdos que son armados inicialmente y más tarde re-construidos en actos de recuerdo (Beltrán-Jaimes, Moreno-López, Polo-Díaz, Zapata-Zabala y Acosta-Barreto, 2012), además, los recuerdos emocionales interrumpen el funcionamiento de la estructura de meta actual, colocando el sistema cognitivo en un estado de preparación para el cambio, por lo tanto, los recuerdos emocionales podrían reinstaurar señales (pasadas) para la acción (Conway y Pleydell-Pearce, 2000). Durante la práctica físico-deportiva, son muchas las metas a las que se ven enfrentados los participantes, las cuales no siempre pueden ser reguladas a nivel personal, como es el caso mayoritario de la formación universitaria o escolar, que se encuentran establecidas en forma estandarizada por las instituciones educativas, sucediendo que exista una considerable discrepancia entre la valoración del estado ideal y el estado actual de la meta (Conway, 2005). Entonces, se producen emociones negativas para el bienestar subjetivo ante los fracasos en el logro de los objetivos y expectativas.

\section{Errores y dificultades en el desempeño motriz}

En las tareas que implican una interacción táctil, se ha detectado que algunos participantes suelen percibir el miedo, ya que por equivocación podrían 
tocar una parte íntima del alumnado (Canales-Lacruz y Rey-Cao, 2014). Esta atribución emocional fue identificada tanto en hombres como en mujeres. Esta situación también se ha vinculado a la falta de experiencias motrices en torno a las tareas desarrolladas, señalando que si los/as participantes tuvieran más oportunidades de participar en ese tipo de actividades, los resultados podrían ser mejores y se reduciría la intimidación sentida al acercarse a sus compañeros o compañeras y demostrar afectividad (Pires de Sousa y Caramaschi, 2011). Cabe destacar, que en esta situación el miedo asumiría un fuerte carácter moral (Mujica, Inostroza y Orellana, 2018; Scheler, 2005), orientado hacia la responsabilidad intersubjetiva. Asimismo, se detecta un patrón común que se resume en miedo a fallar o equivocarse en las tareas motrices en otros estudios (Mujica et al., 2016; Mujica, Orellana y Concha, 2018; Mujica-Johnson y Jiménez, 2019; Ruiz-Sánchez, Gómez-López y Granero-Gallegos, 2017).

En cuanto a la exhibición corporal, se aprecia que es socialmente muy aceptada, promovida y celebrada en la cultura física y deportiva. Sin embargo, si la acción que se debe exhibir es muy exigente para la persona debido a que su capacidad de respuesta no es coherente a ella, ya que será desarrollada con errores producirá miedo, como es el caso de una alumna de EF que fue seleccionada por el docente para ser modelo en la recepción del balón tras un saque. En concreto, cuando no se sentía preparada para cumplir ese rol y tampoco deseaba que el alumnado la observara haciendo algo que no ha aprendido bien, lo que describió como una situación horrible debido a que percibía que hacía el ridículo (Monforte y Pérez-Samaniego, 2017). También esta situación se ha manifestado en las actividades de expresión corporal, en donde se ha identificado que los/as participantes sienten miedo de hacer tonterías (equivocarse) frente a sus compañeros/as, aludiendo la falta de confianza con los observadores (Rodríguez y Rocu, 2016).

El lenguaje corporal, por medio de la postura, los gestos, las miradas, expresa las emociones de cada ser y tiene un valor importantísimo en la vida de las personas, ya que el cuerpo es la expresión de la identidad, por lo que las personas que dirigen la práctica motriz tienen que tomar consciencia de la dificultad que resulta exhibirse en público, ya que no se tiene un cuerpo, sino que se es un cuerpo, se es una realidad corporal (Toro, 2017), por lo tanto, no se exhibe un cuerpo ajeno en acción, sino que la persona se exhibe en acción. A esta situación hay que agregarle un sentido subjetivo más, referido al miedo que genera el enojo del docente de EF ante el error de sus alumnas, el cual es expresado con frases como “¿me estás tomando el pelo?” o "parezco disco rayado", demostrando su malestar por el desempeño motriz de sus aprendices (Monforte y Pérez-Samaniego, 2017). Asimismo, el miedo se ha asociado al lenguaje verbal, en concreto, en el contexto español. Precisamente, cuando el alumnado ha participado de clases de EF realizadas en un segundo idioma, denominado método AICLE, debido a que significaría una gran dificultad para los aprendices (Salvador-García et al., 2018). En estos sentidos, es fundamental la empatía del profesorado para comprender el temor de su alumnado.

\section{Daño y sufrimiento corporal}

La práctica de actividad física y deportiva tiene diferentes manifestaciones, con su respectiva lógica interna, existiendo las que demandan una interacción de contacto, como en la expresión corporal, lo que es valorado como un aspecto amenazante desde la perspectiva de género, ya que los hombres perciben miedo de dañar a sus compañeras. Esta atribución emocional ha sido asociada a un estereotipo de debilidad que asignan los hombres a las mujeres (Canales-Lacruz y Rey-Cao, 2014). Esta concepción de género se manifiesta en la práctica físico-deportiva como una construcción socio-cultural, en donde la masculinidad ocupa una posición hegemónica (Vidiella, Herraiz, Hernández y Sancho, 2010), lo cual es señalado como un aspecto que molesta al género femenino, ya que promueve la desigualdad social. El sentido de generar un daño a la otra persona que participa de la actividad, también se manifiesta en la danza, lo cual dificulta el afrontamiento de la tarea, ya que existe miedo a equivocarse y pisar al compañero de baile (Pires de Sousa y Caramaschi, 2011).

En la práctica deportiva, en EF superior y EF secundaria, también, se ha identificado el miedo a sufrir un accidente ante los riesgos que genera el Parkour (Fernández-Río y Suarez, 2014), el fútbol (Da Silva, 
Richter y Pinto, 2017), Dodgeball (Lee y Kim, 2012), el voleibol (Canales-Lacruz y Pina-Blanco, 2014; Monforte y Pérez-Samaniego, 2017), la Natación (Sato, Ellison y Eckert, 2019), los deportes extremos (Brymer y Schweitzer, 2012), el Ciclismo (Barker et al., 2019). Otra barrera para la actividad física en la etapa infantil asociada al daño corporal es atribuida a la percepción de los padres. Precisamente, porque temen que sus hijos se encuentren con personas extrañas que tengan malas intenciones en entornos comunitarios y escolares (Findholt, Michael, Jerofke y Brogoitti, 2011). Entonces, el miedo que sienten las personas en este contexto deportivo cumple la función de dirigir su atención a protegerse y buscar la forma de afrontar su defensa (López, 2016), ya que la persona se siente amenazada, por lo tanto, quien dirige la actividad motriz, tendrá la posibilidad de ayudar con acciones basadas en la empatía.

En el ámbito se la salud pública, la práctica de actividad física suele ser prescita como un aspecto importante para la prevención y rehabilitación de los pacientes. No obstante, se ha identificado el miedo como una emoción que actuaría como una barrera para la práctica de actividad física. En concreto, por el miedo a las lesiones, al fracaso o al dolor en pacientes con espondiloartritis, osteoporosis, lesión de medula espinal, osteoartritis de rodilla, obesidad, VIH y rotura del ligamento cruzado anterior (Curbelo et al., 2017; Gay, Eschalier et al., 2018; McVeigh y Pack, 2015; Smith, Papathomas, Martin, Latimer-Cheung, 2013; Piana et al., 2013; Vader et al., 2017; Ziebart et al., 2018).

\section{Discursos culturales}

Un estudio en EF con alumnado que tenía espectro autista logró revelar que, en los corredores, antes de la sesión de la asignatura, dichos estudiantes se agobian y sienten miedo ante el caos o desorden que se produce (Lamb, Firbank y Aldous, 2014). Por otro lado, los discursos de masculinidad hegemónica y raza en contexto de EF y deportes han suscitado temor a la discriminación (Beltrán-Carrillo et al., 2016; Forde, 2013; Stewart et al., 2019; Vidiella et al., 2010). Así, el miedo impulsaría a los hombres a mantener una conducta representativa de una orientación heterosexual, evitando de esta forma ser humillados, ya que los actos homofóbicos se producen en diferentes espacios escolares y deportivos (Stewart et al., 2019; Vidiella et al., 2010). Esta identificación con estereotipos sociales masculinos y femeninos son reconocidos tradicionalmente en el ámbito físico-deportivo, atribuyendo al hombre tareas que demandan fuerza, potencia y competitividad (Hellín et al., 2006).

En términos similares, otro estudio señala que el miedo es una de las principales emociones percibidas por el alumnado que es víctima de acoso escolar, el cual incluso, muchas veces, le impide comunicar la problemática a otras personas. En este sentido, dicho estudio revela que los acosadores tienen diversas estrategias para lograr que la intimidación no sea revelado al docente y se mantenga en silencio entre compañeros (O'Connor y Graber, 2014). Aquellas realidades, marcadas por la discriminación y la violencia, demuestran la compleja situación de los juicios sociales y su influencia en la emoción del miedo. Por consiguiente, esta emoción condiciona las posibilidades de acción que tienen las personas en el contexto estudiado, teniendo que decidir si se desenvuelven como ellos desean, enfrentándose a la violencia ejercida por el ambiente o se someten por medio de la adaptación a los patrones culturales del entorno. En este sentido, los estudios revelan en forma clara la falta de respeto hacia la dignidad humana (Kant, 1996; Michelini, 2010) y, en consecuencia, a los derechos humanos (Habermas, 2010).

\section{CONCLUSIONES}

En respuesta al objetivo de esta revisión, se concluye que el miedo en la EF y el deporte responde a una valoración cognitiva que surge de la interacción simbólica-emocional con el entorno, de modo que el contexto sociocultural es fundamental para comprender los factores que lo suscitan. Entonces, el miedo está asociado a lesiones, sufrimiento, errores y dificultades motrices, percepción de la incompetencia motriz y discursos culturales. Además, en parte, el temor se potencia con motivos externos, como la falta de empatía por parte del entorno. Por esa razón, es fundamental que los educadores o técnicos de deportes se encuentren preparados para prevenir dichas situaciones y actuar con comprensión del fenómeno emocional. En cuanto a los discursos culturales que producen malestar psicosocial, es posible concluir que la masculinidad hegemónica y las diferencias raciales suscitan miedo en los contextos estudiados. 
Por otra parte, como estrategia para prevenir el miedo, se reconoce que la adaptación de las tareas motrices al desempeño motriz de cada sujeto contribuiría a prevenir la percepción del miedo al momento de afrontar dichos desafíos pedagógicos. En efecto, por medio de estas adaptaciones se buscaría reducir las expectativas de fracaso y los errores motrices. Sin embargo, se comprende que en una proporción adecuada dicha emoción es importante para activar la atención y protección de las personas, de modo que en ningún caso se debe pretender excluirla del proceso educativo. Por esta razón, algo importante sería contribuir a que las personas puedan superar en gran parte los motivos que producen su miedo y, de esta forma, sentirlo de manera más moderada, sobre todo si se trabaja en contextos pedagógicos seguros y propicios para el aprendizaje. Asimismo, dado que es una emoción que cumple funciones adaptativas importantes, es de vital importancia que se presente en los momentos precisos, por ejemplo, cuando el alumnado debe superar sus límites motrices o debe resguardar su integridad ante una situación inesperada.

Hay amenazas culturales que suscitan miedo y serían reflejo de una sociedad carente de buenos valores ético-morales, en concreto, marcada por una falta de respeto a la dignidad humana. Es común que en estos ambientes se generen situaciones estresantes para las personas por motivos totalmente ajenos al proceso individual de aprendizaje o desarrollo motriz. Por esta razón, dado que los procesos de EF, actividad física y deporte son generalmente situaciones de aprendizaje colectivo, es que los temores se presentan como un buen indicador para reconocer los aspectos morales que deben ser mejorados. En este sentido, el cuerpo técnico o el profesorado requiere evaluar las emociones del alumnado para apoyar al alumnado que percibe miedo y, en el caso de que sea provocado por terceras personas, contribuir al desarrollo moral de quienes suscitan miedo en sus pares.

Finalmente, se aprecia la necesidad de promover acciones pedagógicas de educación afectiva-moral que ayude a tratar el miedo de las personas, así como la investigación y reflexión sobre las ideologías que se encuentran implícitas en estos entornos, con el fin de prevenir experiencias innecesarias de malestar subjetivo.

\section{REFERENCIAS}

Barker, D., Nyberg, G. y Larsson, H. (2019). Joy, fear and resignation: investigating emotions in physical education using a symbolic interactionist approach. Sport, Education and Society, 1-17. https://doi.org/10.1080 /13573322.2019.1672148

Beltrán-Carrillo, V., Devís-Devís, J. y Peiró-Velert, C. (2016). The influence of body discourses on adolescents' (non) participation in physical activity. Sport, Education and Society, 23(3), 257-269. https://doi.org/10.1080/135 73322.2016.1178109

Beltrán-Jaimes, J., Moreno-López, N., Polo-Díaz, N., Zapata-Zabala, M. y Acosta-Barreto, M. (2012). Memoria autobiográfica: un sistema funcionalmente definido. International Journal of Psychological Research, 5(2), 108-123. https://doi.org/10.21500/20112084.742

Bolaños, F. (2016). El estudio socio-histórico de las emociones y los sentimientos en las ciencias sociales del siglo xx. Revista de estudios sociales, 55(35), 178-191. https://doi.org/10.7440/res55.2016.12

Boyano, J. (2012). Bases neuropsicológicas de la memoria autobiográfica. Revista Chilena de Neuropsicología, 7(3), 98-101. doi: 10.5839/rcnp.2012.0703.01

Canales-Lacruz, I., y Rey-Cao, A. (2014). Diferencias de género percibidas por el alumnado en la interacción visual y táctil de las tareas de expresión corporal. Movimento, 20(1), 169-192.

Canales-Lacruz, I., y Pina-Blanco, I. (2014). El miedo al contacto en el voleibol. Percepciones del alumnado de Educación Física. Ágora para la Educación Física y el Deporte, 16(2), 122-136.

Consuegra, N. (2010). Diccionario de psicología. Bogotá: Ecoe.

Conway, M. (2005). Memory and the self. Journal of Memory and Language, 53, 594-628. https://doi. org/10.1016/j.jml.2005.08.005

Conway, M. y Pleydell-Pearce, C. (2000). The construction of autobiographical Memories in the self-memory system. Psychological Review, 107(2), 261-288. https:// doi.org/10.1037/0033-295X.107.2.261

Curbelo, R., Zarco, P., Almodóvar, R., Flórez, M. y Carmona Ortells, L. (2017). Barreras y facilitadores para la práctica del ejercicio físico en pacientes con espondiloartritis: estudio cualitativo de grupos focales (EJES-3D). Reumatología Clínica, 13(2), 91-96. doi:10.1016/j. reuma.2016.03.004 
Da Silva, J., Richter, A. y Pinto, F. (2017). O sentido do futebol nas aulas de educação física. Movimento, 23(4), 13951406. https://doi.org/10.22456/1982-8918.64681

Fernández-Río, J. y Suarez, C. (2014). Feasibility and students' preliminary views on parkour in a group of primary school children. Physical Education and Sport Pedagogy, 21(3), 281-294. https://doi.org/10.1080/1 7408989.2014.946008

Findholt, N., Michael, Y., Jerofke, L. y Brogoitti, V. (2011). Environmental Influences on Children's Physical Activity and Eating Habits in a Rural Oregon County. American Journal of Health Promotion, 26(2), 74-85. https:// doi.org/10.4278\%2Fajhp.100622-QUAL-210

Forde, S. (2013). Fear and loathing in Lesotho: An autoethnographic analysis of sport for development and peace. International Review for the Sociology of Sport, 50(8), 958-973. https://doi. org/10.1177\%2F1012690213501916

Gay, C., Eschalier, B., Levyckyj, C., Bonnin, A. y Coudeyre, E. (2018). Étude qualitative des freins et des éléments de motivation à la pratique d'une activité physique régulière auprès d'une population de gonarthrosiques. Revue Du Rhumatisme, 85(6), 550-556. https://doi. org/10.1016/j.rhum.2017.11.003

González, A. y González, C. (2010). Educación física desde la corporeidad y la motricidad. Hacia la promoción de la salud, 15(2), 173-187.

González, F. (2002). Sujeto y subjetividad: una aproximación histórico-cultural. México: Thomson.

González, F. (2008). Subjetividad social, sujeto y representaciones sociales. Revista Diversitas, 4(2), 225-243.

González, F. (2009). La significación de Vygotski para la consideración de lo afectivo en la Educación: Las bases para la cuestión de la subjetividad. Revista Electrónica Actualidades Investigativas en Educación, 9, 1-24. doi: 10.15517/aie.v9i4.9519

Habermas, J. (2010). El concepto de la dignidad humana y la utopía realista de los derechos humanos. Diánoia, 55(64), 3-25. https://doi.org/10.21898/dia. v55i64.218

Hellín, P., Moreno, J. y Rodríguez, P. (2006). Relación de la competencia motriz percibida con la práctica físico-deportiva. Revista de Psicología del Deporte, 15(2), 219-231.

Huber, A. (2000). El miedo. Madrid: Acento.
Hurtado, D. (2008). Corporeidad y motricidad. Una forma de mirar los saberes del cuerpo. Revista Educação \& Sociedade, 29(102), 119-136. https://doi.org/10.1590/ S0101-73302008000100007

Husserl, E. (1962). Ideas relativas a una fenomenología pura y una filosofía fenomenológica. México: Fondo de Cultura Económica.

Kant, I. (1996). Fundamentación de la metafísica de las costumbres. México: Porrúa.

Lamb, P., Firbank, D. y Aldous, D. (2014). Capturing the world of physical education through the eyes of children with autism spectrum disorders. Sport, Education and Society, 21(5), 698-722. https://doi.org/10.1080/ 13573322.2014 .941794

Lazarus, R. (2000). Estrés y emoción. Manejo e implicaciones en nuestra salud. Bilbao: Desclée de Brouwer.

Lee, B. y Kim, D. (2012). The context experiencing for girl students in fear and anxiety in physical education class and its educational meaning. Secondary Education Research, 60(3), 781-803.

Leisterer, S. y Jekauc, D. (2019). Students' emotional experience in Physical Education. A qualitative study for new theoretical insights. Sports, 7, 1-15. https://doi. org/10.3390/sports7010010

López, B. (2016). Aprendizaje emocionante. Neurociencia para el aula. Madrid: Biblioteca Innovación Educativa.

McVeigh, F. y Pack, S. (2015). An Exploration of Sports Rehabilitators' and Athletic Rehabilitation Therapists' Views on Fear of Reinjury after Anterior Cruciate Ligament Reconstruction. Journal of Sport Rehabilitation, 24(2), 140- 150. https://doi.org/10.1123/ jsr.2014-0127

Merello, M. y Oliva, P. (2012). La obesidad y su relación con las patologías periodontales: una revisión narrativa. International Journal of Odontostomatology, 6(2), 235-239. https://doi.org/10.4067/ s0718-381x2012000200019

Michelini, D. (2010). Dignidad humana en Kant y Habermas. Estudios de filosofía práctica e historia de las ideas, 12(1), 41-49.

Monforte, J. y Pérez-Samaniego, V. (2017). El miedo en educación física: una historia reconocible. Movimento, 23(1), 85-99. https://doi.org/10.22456/1982-8918.71272

Mora, F. (2017). Neuroeducación. Solo se puede aprender aquello que se ama. Madrid: Alianza. 
Mujica, F. (2020). El término Educación Física en la posmodernidad: contribución de algunas perspectivas fenomenológicas. Retos. Nuevas Tendencias en Educación Física, Deportes y Recreación, 38, 1-7.

Mujica, F., Orellana, N., Aránguiz, H. y González, H. (2016). Atribución emocional de escolares de sexto año básico en la asignatura de Educación Física y Salud. Educación Física y Ciencia, 18(2), 1-6.

Mujica, F., Inostroza, C. y Orellana, N. (2018). Educar las emociones con un sentido pedagógico: Un aporte a la justicia social. Revista Internacional de Educación para la Justicia Social, 7(2), 113-127. https://doi. org/10.15366/riejs2018.7.2.007

Mujica-Johnson, F. y Jiménez, A. (2019). Percepción emocional en la asignatura de Baloncesto de estudiantes del grado en Ciencias del Deporte: Estudio piloto. Cuadernos de Psicología del Deporte, 19(2), 152-166.

Mujica, F., Orellana, N. y Concha, R. (2017). Emociones en la clase de Educación Física: revisión narrativa (2010-2016). Ágora para la Educación Física y el Deporte, 19(1), 119-134. https://doi.org/10.24197/ aefd.1.2017.119-134

Mujica, F., Orellana, N. y Concha, R. (2018). Atribución emocional en el taller de Baloncesto escolar de una escuela pública en Chile: análisis de contenido. Cuadernos de Psicología del Deporte, 18(1), 31-42.

O'Connor, J. y Graber, K. (2014). Sixth-Grade Physical Education: An Acculturation of Bullying and Fear. Research Quarterly for Exercise and Sport, 85(3), 398- 408. https://doi.org/10.1080/02701367.2014.930403

Piana, N., Battistini, D., Urbani, L., Romani, G., Fatone, C., Pazzagli, C., ... y De Feo, P. (2013). Multidisciplinary lifestyle intervention in the obese: Its impact on patients' perception of the disease, food and physical exercise. Nutrition, Metabolism and Cardiovascular Diseases, 23(4), 337-343. https://doi.org/10.1016/j. numecd.2011.12.008

Pires de Sousa, N. y Caramaschi, S. (2011). Contrato corporal entre adolescentes a través da dança de salão na escola. Motriz: Revista de Educação Física, 17(4), 618-629. https://doi.org/10.1590/ S1980-65742011000400006

Rebollo, M. y Hornillo, I. (2010). Perspectiva emocional en la construcción de la identidad en contextos educativos: discursos y conflictos emocionales. Revista de Educación, 353, 235-263.
Rodríguez, A. y Rocu, P. (2016). Emociones percibidas a través de las primeras experiencias de expresión corporal: estudio de casos en secundaria. EmásF. Revista Digital de Educación Física, 40, 134-157.

Rother, E. (2007). Revisión sistemática x revisión narrativa. Acta Paulista de Enfermagem, 20(2), 9-10.

Ruiz, L. y Graupera, J. (2003). Competencia motriz y género entre los escolares españoles. Revista internacional de Medicina y Ciencias de la Actividad Física y el Deporte, 3(10), 101-111.

Ruiz-Sánchez, V., Gómez-López. M., Granero-Gallegos, A. y González-Hernández, J. (2017). Relación del clima motivacional y miedo al fallo en jugadores de alto rendimiento en balonmano. Cuadernos de Psicología del Deporte, 17(3), 55-64.

Sato, T., Ellison, D. y Eckert, K. (2019). African American pre-service physical education teachers' learning about aquatic courses. European Physical Education Review, 25(3), 778-795. https:// doi.org/10.1177\%2F1356336X18774760

Salvador-García, C., Chiva-Bartoll, Ó. y Vergaz, J. (2018). Percepción del alumnado sobre el uso del método AICLE en Educación Física: estudio de caso. Retos. Nuevas Tendencias en Educación Física, Deportes y Recreación, 33, 138-142.

Scheler, M. (2005). Esencia y formas de la simpatía. Sígueme: Salamanca.

Smith, B., Papathomas, A., Martin, K. y Latimer-Cheung, A. (2013). Understanding physical activity in spinal cord injury rehabilitation: translating and communicating research through stories. Disability and Rehabilitation, 35(24), 2046- 2055. https://doi.org/10.3109/096382 88.2013.805821

Stein, E. (2007). La estructura de la persona humana. Madrid: BAC.

Stewart, S., Gray, S., Kelly, J. y Maclsaac, S. (2019). Investigating the development of masculine identities in physical education. Sport, Education and Society, 1- 15. https://doi.org/10.1080/13573322.2019.1654447

Taylor, S. y Bogdan, R. (2009). Introducción a los métodos cualitativos de investigación. Barcelona: Paidós.

Toro, J. (2017). Educar con co-razón. Bilbao: Desclée de Brouwer. 
Torrents, C., Mateu, M., Planas, A. y Dinusôva, M. Posibilidades de las tareas de expresión corporal para suscitar emociones en el alumnado. Revista de Psicología del Deporte, 20(2), 401-412.

Vader, K., Simonik, A., Ellis, D., Kesbian, D., Leung, P., Jachyra, P., ... y O'Brien, K. K. (2017). Perceptions of "physical activity" and "exercise" among people living with HIV: A qualitative study. International Journal of Therapy and Rehabilitation, 24(11), 473-482. https:// doi.org/10.12968/ijtr.2017.24.11.473

Vidiella, J., Herraiz, F., Hernández, F. y Sancho, J. (2010). Masculinidad hegemónica, deporte y actividad física. Movimento, 16(4), 93-115. https://doi. org/10.22456/1982-8918.15031
Zaccagnini, J. (2008). La comprensión de la emoción. Una perspectiva psicológica. En M. Jiménez (ed.), Educación emocional y convivencia en el aula (pp. 31-84). Madrid: Ministerio de Educación, Política Social y Deporte.

Ziebart, C., McArthur, C., Lee, L., Papaioannou, A., Laprade, J., Cheung, A. M., ... y Giangregorio, L. (2018). "Left to my own devices, I don't know": using theory and patient-reported barriers to move from physical activity recommendations to practice. Osteoporosis International, 29(5), 1081-1091. https://doi.org/10.1007/ s00198-018-4390-3 